\section{Journal Watch}

Neil Wigglesworth' and Deborah Xuereb²
Journal of Infection Prevention

2015, Vol. 16(5) 233-235

DOI: $10.1177 / 1757177415599833$

(C) The Author(s) 2015

Reprints and permissions:

sagepub.co.uk/journalsPermissions.nav

jip.sagepub.com
The journal watch feature is provided as a service to our readers. The intention is to highlight new research and other developments in infection prevention and control and related fields, published elsewhere. A brief description of each article and its main findings is given here; readers are encouraged to refer to the full published article for the details of the work. The authors and the editorial management group would welcome feedback and recommendations for articles to feature in this column; for comments and recommendations please contact neil.wigglesworth@ gmail.com or editor@ips.uk.net.

The articles in this Journal Watch are an eclectic collection; the authors are grateful to ex-IPS President Martin Kiernan for introducing them to the joy of RSS feeds and feed readers (if you don't have one but want to keep up with what's being published, get one!). The articles range from clinical to quite abstract and from multi-resistant organisms to surveillance definitions, through the behaviours of doctors and the effect of economics on infection.

The first article in this issue comes from the Journal of Hospital Infection and looks at an issue that has rarely been studied: healthcare infection risk and socioeconomic deprivation.

Packer SJ, Cairns S, Robertson C et al. (2015) Determining the effect of social deprivation on the prevalence of healthcare-associated infections in acute hospitals: A multivariate analysis of a linked data set. Journal of Hospital Infection; DOI: 10.1016/j.jhin.2015.06.014.

The authors of this major study have done something I've previously discussed wanting to do with colleagues in public health, examined the link between socioeconomic deprivation and healthcare-associated infection (HCAI). As these authors point out, the link between ill health, poor health outcomes and deprivation is clearly established and can't simply be explained by health behaviours. We know that being deprived takes both years off your life, as well as life (wellbeing) out of your years. This is the first study to examine this phenomenon across the range of HCAI and it's both welcome and overdue. The study used linkage of big datasets; namely the 2012 Scottish Point Prevalence Survey of HCAI (and antimicrobial use) and the Scottish Index of Multiple Deprivation (SIMD). The authors don't elaborate on it, but I'm assuming this is similar if not identical to the Index of Multiple Deprivation used in England and Wales. The details of how the linking was done are in the paper and can be followed by anyone seeking to replicate the method; the key point is that the SIMD is a deprivation 'score' for the area where the patient lives, not for the individual (the authors rightly warn that these data are subject to the 'ecological fallacy'). The study used univariable and multivariable analysis to examine the relationship between HCAI, deprivation and other possible factors such as demographics and co-morbidities. The results are interesting and perhaps surprising at face value. There are two headlines: first that they found no association between HCAI in general and SIMD, but they did find one when the patients were having surgery. It appears from their results that being deprived means you are more likely to end up in hospital, but once there, not generally more likely to get an infection. In discussion they suggest that once in hospital everyone gets the same standard of care and management and this negates the effect of deprivation; in addition, they suggest that perhaps deprivation has an impact on the epidemiology of Staphylococcus aureus which could explain the results in patients having surgery. This is an important piece of work and should be followed up by further studies to confirm or refute its findings. Finally and slightly flippantly, it appears our Scottish colleagues have conceded the battle on acronyms, as the article used HCAI and not the Scottish HAI!

The issue of whether glove wearing has an adverse effect on hand hygiene in healthcare is a hot topic, indeed the Editor of this journal and the President of IPS have published work on this very subject recently with other colleagues (Wilson et al., 2015). Our second article adds evidence to the view that there is such a detrimental effect;

\footnotetext{
'Infection Prevention and Control, Guy's and St Thomas' NHS Foundation Trust, Westminster Bridge Road, London SEI 7EH, UK

2Infection Control Unit, Mater Dei Hospital, Malta, UK

Corresponding author:

Neil Wigglesworth, Deputy Director, Infection Prevention and Control, Guy's and St Thomas' NHS Foundation Trust, Westminster Bridge Road, London SEI 7EH, UK.

Email: neil.wigglesworth@gstt.nhs.uk
} 
Cusini A, Nydegger D, Kaspar T et al. (2015) Improved hand hygiene compliance after eliminating mandatory glove use from contact precautions - Is less more? American Journal of Infection Control; DOI: 10.1016/j. ajic.2015.05.019.

This is a very courageous piece of work; following on from previous data that suggested that using gloves routinely as part of contact precautions led to poor hand hygiene compliance, this hospital removed the requirement for gloves unless indicated by standard precautions. This approach took them firmly outside current guidance and I'd be surprised if any UK healthcare organisation would be so brave. The article reports hand hygiene observations from before the change and afterwards and includes patients on contact precautions as well as whole hospital hand hygiene compliance data. The methods for observing hand hygiene compliance are quite standard and most infection prevention teams would recognise them, although the attention to reliability and rigour seem very high (e.g. annual refreshers for observers). In their defence the authors point out, rightly, that there isn't much evidence for routine glove use. The results are very interesting; the authors use data from 2009 and 2012 (the change took place in 2011) - they explain this as a resource issue (presumably for observing hand hygiene in the care of patients on contact precautions). Overall, in their large hospital, in Switzerland, hand hygiene compliance was worse in the care of patients on contact precautions before the change (2009) and better after the change (2012). In both years this is in comparison to the whole hospital data. This does suggest that eliminating mandatory gloving improved hand hygiene compliance significantly. There is some detail; the improvement was marked in Moment 1 and Moment 2 (WHO), whereas Moment 4 was consistently high throughout in contact precautions. This suggests that routine gloving makes hand hygiene less likely on the approach to a patient and when it's needed prior to an aseptic procedure and this does seem likely at face value. There are two comments worth noting and making; first to note that Clostridium difficile was specifically excluded from the study (but it doesn't say if those patients were excluded from the intervention) and the question it doesn't answer is: which is best for preventing infection, routine gloves or increased hand hygiene compliance?

Continuing the theme of hand hygiene compliance, the next publication from BMJ Quality and Safety, looks with some insight and sympathy at the thorny issue of doctors' hand hygiene behaviours.

\section{Redelmeier DA and Shafir E (2015) Why even good physicians do not wash their hands? BMJ Quality \& Safety Online First; DOI: 10.1136/bmjqs-2015-004319.}

This viewpoint delves into the behavioural reasons why doctors do not clean their hands as often as other healthcare professionals. Initially the reader gets a feeling that this paper will take on an apologetic approach, but on reading further, the authors put forward several convincing explanations why doctors may not clean their hands. The authors suggest that the circumstances surrounding doctors in hospitals are conducive to failures of hand hygiene. The authors (who seem not to be IPC professionals as they constantly refer to hand hygiene as 'hand washing') suggest that the reasons why doctors fail to clean their hands frequently, fall into three main categories: affective (emotional); cognitive; and social factors. When doctors actually clean their hands, there is little positive reinforcement and little feeling of accomplishment. The distant connection to outcomes means that failure to clean hands does not raise the worry of a possible life-threatening mistake. Hand hygiene may not be high on a doctor's priority list, since it does not directly relieve pain or other form of patient suffering. The timing when hand hygiene is required often coincides with moments when the doctor's attention is focused onto new, often demanding situations. Also, hand hygiene is seen as a monotonous and repetitive procedure. The paper also noted several social factors impacting on doctors' hand hygiene behaviours. Hand hygiene holds insufficient prestige for doctors who are 'unlikely to pride themselves for a hand hygiene award'! Hand hygiene auditing is sometimes a blameful and embarrassing negative feedback exercise. Lack of hand hygiene is difficult to monitor with violations largely invisible. When there is lack of hand hygiene among doctors, it is easy for younger doctors to get accustomed to this as the norm. The authors challenge the integrity of hand hygiene audits, arguing that adherence among nurses is possibly overestimated due to the Hawthorne effect, since auditors are easily recognized. They conclude by giving a few examples of how we can translate this behavioural knowledge to better hand hygiene improvement programmes. This article makes a good contribution to published literature on doctors' hand hygiene behaviours and puts forward thought provoking and at times controversial points.

Many in the infection prevention community are struggling with how to cope with the increasing burden of carbapenem resistant Enterobacteriaceae (CRE), lessons from around the world may help us, such as those in this next paper, from Singapore.

Ling ML, Tee YM, Tan SG et al. (2015) Risk factors for acquisition of carbapenem resistant Enterobacteriaceae in an acute tertiary care hospital in Singapore. Antimicrobial Resistance and Infection Control 4: 26. http://www.aricjournal.com/content/4/1/26.

The global increase in CRE is a current challenge to IC professionals. Identifying risk factors for carriage and acquisition could help us develop risk-based screening to limit the spread of CRE. This matched case-control study from Singapore sought to identifying risk factors for 
carriage of CRE among inpatients treated in a large tertiary acute care hospital. Two hundred and three cases in whom CRE were isolated from clinical cultures from any source over a period of 2 years, were matched with the same number of randomly selected controls. PCR was used to identify CRE types. The most common CRE type seen was NDM-1, followed by KPC and very few OXA-48. As in other studies, the researchers found that exposure to antibiotics, especially carbapenems and fluoroquinolones, admission to ICU, presence of indwelling devices such as central line, urinary catheter, endotracheal tube and enteral feeding tube, increased risk for CRE. Exposure to penicillin, glycopeptides and presence of central line device were independently associated with CRE infection. The mean total length of hospital stay was impressively longer in CRE cases (48.0 days) than controls ( 3.9 days, $P<0.001)$ possibly explained by the fact that cases had a higher probability to have co-morbidities. Indeed haematology patients and those with immunodeficiency were more likely to have CRE. Cases had approximately 3.5 times odds of fatality compared to controls, highlighting the impact on patients.

The study had two major limitations: the small sample size which made the study underpowered; and the fact that no admission or exit screening was done made it difficult to establish whether patients acquired CRE during their hospital stay or had it on admission. Thus the researcher could not correlate the link between the risk factors and acquisition; indeed the researchers recommend targeted screening strategies to identify patients colonised with CRE at the time of admission as a component of a CRE reduction program. Overall this study highlights the importance of safe clinical practices especially in the care of devices as well as the significance of an antimicrobial stewardship programme. Although this study is very context-specific, it still draws out some risk factors which could have applicability elsewhere, especially in areas were CRE are endemic.

This last publication is one of the occasional series of 'and finally' pieces and has a few things to tell us about the difficulties of surveillance in a world of reporting and targets.

\footnotetext{
Allen-Bridson K, Pollock D and Gould CV (2015) Promoting prevention through meaningful measures: Improving the Centers for Disease Control and Prevention's National Healthcare Safety Network urinary tract infection surveillance definitions. American Journal of Infection Control; doi: 10.1016/j.ajic.2015.06.006.
}

This piece is a commentary on the revision of the Centers for Disease Control and Prevention (CDC) surveillance definition for urinary tract infection (UTI). I've included it for a number of reasons; first that, in the UK, with the honourable exception of Scotland, surveillance of UTI has been put firmly in the 'too difficult' box. In England we have the highly unscientific but arguably 'better than nothing' Safety Thermometer and to the best of my knowledge there's nothing in Wales or Northern Ireland (or the Republic). In the US they've been doing surveillance a lot longer of course and this is only the latest iteration of their definition. More reasons to follow, but first the nuts and bolts of the article; the definition has been arrived at by expert consensus and the changes are the removal of some of the less specific criteria (such as elements of urinalysis and lower organism counts) and also of fungal isolates as a criterion for reporting. Each of these changes is given a clear and well thought out rationale and seems reasonable, but there is an underlying theme. This theme is the second reason that this is an interesting article. The changes that have been made are ostensibly in the cause of specificity (and reasonably so) but equally they reduce the sensitivity of the surveillance and there is a reason for this in the text. It seems clear that aspects of mandatory reporting which is linked to reimbursement have had some impact. The authors specifically cite the 2012 change to reporting thus '...the inclusion of catheter-associated urinary tract infections (CAUTIs) in the Centers for Medicare and Medicaid Services' Inpatient Quality Reporting Program in 2012 heightened the challenges to the definitions by many professionals involved in infection prevention'. It seems very clear that because these infections are subject to formal reporting, there is more anxiety about the definition.

If this collection of papers, all from the very latest 'in press' publications, has converted you to the joy of RSS feeds, then please let us know what gems of articles you discover. The articles that excite you are likely to excite others and we can share them via this regular column; all contributions welcome! (More about RSS feeds at https:// en.wikipedia.org/wiki/RSS.)

\section{Reference}

Wilson J, Prieto J, Singleton J, et al. (2015) The misuse and overuse of non-sterile gloves: Application of an audit tool to define the problem Journal of Infection Prevention 16: 24-31. 